\title{
LOS DESACUERDOS JURÍDICOS DESDE LA FILOSOFÍA*
}

\author{
Pau Luque \\ Universidad Federico II de Nápoles
}

\begin{abstract}
RESUMEN. En este trabajo se aborda la cuestión de los desacuerdos jurídicos utilizando las herramientas conceptuales provenientes del debate sobre los denominados "desacuerdos intachables» en filosofía. Un «desacuerdo intachable» es una situación en la que las partes discrepan y ninguna de ellas ha cometido error alguno. Aquí se defenderá una posición intermedia, tanto en el derecho como en la filosofía, según la cual existen casos en que habrá una pluralidad de respuestas y esto no denotará ningún error, de manera que admitirá la posibilidad de «desacuerdos intachables" para esos casos. Mientras que habrá otros casos en los que sí podremos decir que hay una única respuesta correcta y en que, en caso de desacuerdo, una de las partes habrá cometido un error.
\end{abstract}

Palabras clave: desacuerdos intachables, error, irenismo, escepticismo, pluralismo.

\section{Legal Disagreements Viewed from Philosophy}

ABSTRACT. This paper deals with the topic of legal disagreements using the conceptual tools of the debate about the so-called «faultless disagreements» in general philosophy. A «faultless disagreement» is a situation in which two or more agents disagree and none of them has made a mistake. I will defend an intermediate position, both in law and in general philosophy, according to which there are some cases susceptible to a plurality of answers, but this does not involve any error at all. So I will admit the possibility of faultless disagreements at least for those cases, while there are other cases in which we can say that there is just one right answer and that, in case of disagreement, one of the parties will be in error.

Keywords: faultless disagreements, error, irenism, skepticism, pluralism.

* Fecha de recepción: 1 de julio de 2013. Fecha de aceptación: 20 de septiembre de 2013.

Este trabajo fue presentado en diversas sedes. En primer lugar, por orden cronológico, en el marco del ciclo de seminarios del área de Filosofia del dret de la Universitat de Girona el 19 de octubre de 2012; recuerdo preguntas y dudas de J. FerRer, J. Matida y D. M. PAPAYANnis. También fue presentado en el Congreso franco-italiano-español de filosofía del derecho, que tuvo lugar en Milán, en la Universidad Luigi Bocconi el 26 de octubre de 2012; allí me plantearon cuestiones, que yo recuerde, Á. NúÑEZ, P. CHIASSONI, M. NARVÁEZ, A. Sardo, D. M. Papayannis, P. Comanducci, D. Canale, G. Tuzet, L. Duarte d’Almeida, J. J. Moreso y J. FERRER. Por último, fue presentado en el seminario de profesores a tiempo completo del ITAM, en México D. F., el 3 de mayo de 2013; R. VÁzquez, R. Gama, H. G. Bouvier y J. Cerdio me plantearon en esa sede agudas cuestiones. Todas estas personas contribuyeron, con sus observaciones, a que pensara mejor las tesis defendidas en el trabajo y es por ello mismo que quiero agradecer su contribución (si he cometido el error de olvidar alguna intervención en esas sedes, me excuso de antemano). Por otro lado, quisiera también agradecer a un anónimo dictaminador sus perspicaces sugerencias al texto. 


\section{INTRODUCCIÓN}

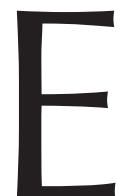

n este trabajo pretendo abordar la cuestión de los desacuerdos jurídicos desde una perspectiva concreta, la de los «faultless disagreements» o «desacuerdos intachables». En primer lugar, reconstruiré el debate, en el seno de la filosofía analítica contemporánea, en torno a la noción de «desacuerdos intachables». En segundo lugar, correlacionaré las posiciones en el debate sobre los «desacuerdos intachables» con dos principales posiciones en el debate iusfilosófico. En tercer lugar, mostraré algunas debilidades de las posiciones mencionadas y propondré una posición «nueva». Por último, a la luz del debate sobre los «desacuerdos intachables», y en el contexto de la discusión sobre los desacuerdos jurídicos, sugeriré la superioridad explicativa de una de las posiciones frente a las otras.

\section{1. «DESACUERDOS INTACHABLES (FAULTLESS DISAGREEMENTS)»}

En filosofía del lenguaje se ha vivido estos últimos años todo un debate acerca de los llamados «faultless disagreements», expresión que aquí será traducida como «desacuerdos intachables». En lo que sigue reconstruiré brevemente este debate.

Es una idea bastante extendida que hay algunas cuestiones o ámbitos sobre los que pueden existir «desacuerdos intachables» (DI, en lo que sigue $)^{1}$. ¿Pero qué quiere decir «desacuerdos intachables»?

«A faultless disagreement is a situation where there is a thinker $A$, a thinker $B$, and a proposition (content of judgment) $p$, such that:

4. $A$ judges (believes that) $p$ and $B$ judges (believes that) not- $p$.

5. Neither $A$ or $B$ has made a mistake (is at fault) $)^{2}$.

Las cuestiones o ámbitos respecto de los cuales se considera que pueden darse estas situaciones son la estética, el gusto o los valores morales ${ }^{3}$.

Así, por ejemplo, A piensa que Paul Auster es mejor que Philip Roth, mientras que $\mathrm{B}$ piensa que Roth es mejor escritor que Auster. O bien $\mathrm{C}$ piensa que es moralmente correcto decir siempre la verdad, mientras que $\mathrm{D}$ afirma que no es moralmente correcto decir siempre la verdad. O bien Riccardo afirma que es mejor el Barolo que el Priorat, mientras que Paolo juzga que el Priorat es mejor vino que el Barolo.

1 Sigo aquí a KÖLBEL, 2003: 53 y ss., y a IACONA, 2008: 287 y ss. Estas cuestiones fueron formuladas originariamente por C. WRIGHT, quien denominó a los DI como «disputes of inclination», en contraste con divergencias en las que la respuesta está objetivamente determinada, WRIGHT, 2001: 47.

2 KölBEL, 2003: 53-54.

3 Soy de los que piensan que hay buenas razones para considerar que la moralidad no está al mismo nivel que la estética o el gusto, y que preguntarse acerca de cuestiones morales no es lo mismo que preguntarse acerca de si es más bueno el bacalao al pil-pil o la «esqueixada de bacallà» o de si la mejor novela de J. CERCAS es Anatomía de un instante o Soldados de Salamina. Pero también es cierto que para quien haya tenido una formación neo-empirista, como es mi caso, la primera intuición pre-teórica tiende a agrupar todos estos ámbitos. No pondré en duda aquí esta premisa y respetaré la disposición de KöLBEL. 
En estos casos nos sentimos inclinados a decir que ninguna de las dos partes ha cometido ningún tipo de error ${ }^{4}$.

La existencia de los DI ha sido replicada en dos sentidos.

Por un lado, el realismo ${ }^{5}$ ha afirmado que los DI son en realidad sólo «desacuerdos», puesto que siempre habrá un error cometido por parte de alguno de los hablantes.

Por otro lado, el relativismo indexical ha afirmado que los DI son en realidad situaciones meramente «intachables», y que no se trata de desacuerdos reales entre las partes.

A continuación detallaré ambos tipos de objeciones, pero, de ser cierto que alguno de los dos lleva razón, entonces no habría espacio para los DI en nuestra semántica estándar (ya sea realista o relativista indexical) ${ }^{6}$.

S: si un sujeto afirma que $p$ y un sujeto afirma que no- $p$, entonces uno de esos sujetos está en un error.

Esto es, por ejemplo, si verificamos que existe un determinado estado de $\cos a s p$, entonces afirmar que no- $p$ denota un error. Y, por tanto, no hay espacio para los DI. El siguiente esquema prueba esto último ${ }^{7}$ :

1 A afirma que $p$ y B afirma que no- $p$

$2 \mathrm{Ni}$ A ni B han cometido un error

$3 p$

4 B está en un error

5 No-p

6 A está en un error

7 No-2
Asunción.

Asunción.

Asunción.

$1,3 \mathrm{~S}$.

2, 3, 4 RAA.

$1,5 \mathrm{~S}$.

2, 2, 6 RAA.

1 es la hipótesis según la cual A y B están en desacuerdo. 2 es la hipótesis que intenta hacer espacio para los DI y nos pide que asumamos que el desacuerdo entre A y B es precisamente un DI. 3 es la asunción que nos permite pasar de 1 a 4 , teniendo en cuenta S. 5 se obtiene a partir de 2, 3 y 4 y la contradicción que aparece entonces queda diluida mediante una reductio ad absurdum que descarta 3.6 se obtiene a partir de $\mathrm{S}$, pues si se verifica no-p y hay uno de los dos agentes que ha cometido un error, entonces A ha cometido un error. 7 es fruto, nuevamente, de una reductio ad absurdum para evitar la contradicción entre 4 y 6 . Así pues, en palabras del propio IACONA, «[f] rom 1-7 it turns out that if $A$ and $B$ disagree it is not the case that neither $A$ nor $B$ is at fault,

${ }^{4}$ De todas formas, que los DI existan en estos ámbitos no quiere decir que todas las disputas en estos ámbitos sean DI. Puede haber algunas discrepancias sobre estas cuestiones que involucren algún tipo de error; por ejemplo, si B ha confundido a Philip Roth con Joseph Roth o con Henry Roth entonces la afirmación según la cual «Philip Roth es mejor escritor que Paul Auster» involucra un error y no podría hablarse de DI en este caso.

5 La etiqueta «realismo» no guarda relación aquí ni con el realismo moral ni con el realismo jurídico, así que para evitar confusiones de ahora en adelante llamaré «realismo semántico» (aun cuando no sea del todo exacto) a esta corriente.

${ }^{6}$ En realidad las posibilidades son más ( $v i d$. KÖLBEL, 2003: 56-57), pero en todo caso son refinamientos de estas dos posiciones, por lo que siguiendo a IACONA, 2008: 290, asumiré que realismo y relativismo indexical ofrecen las objeciones más relevantes a la posibilidad de los DI.

7 IACONA, 2008: 287-288. 
which is classically equivalent to the conclusion that if $A$ and $B$ disagree then either $A$ or $B$ is at fault ${ }^{8}$.

De manera que de tal esquema se sigue «the negation of the claim that there are faultless disagreements, so either there is something wrong with it, or the claim is not true» ${ }^{9}$. Que no hay espacio precisamente para los DI ha sido sostenido desde las dos posiciones diferentes mencionadas anteriormente.

Por un lado, el realismo semántico sostiene que los DI únicamente son «intachables» en apariencia. O A o B ha cometido un error, aun cuando no haya manera de resolver la disputa descubriendo cuál de los dos ha cometido la tacha. Todos los desacuerdos, según el realismo, involucran algún tipo de error por parte de alguno de los agentes.

Por otro lado, el relativismo indexical sostiene que los DI en realidad son únicamente «intachables» y que los desacuerdos sólo lo son en apariencia, puesto que no tienen el mismo objeto. Me explico. Cuando A afirma que «x es sabroso» y B afirma que «x no es sabroso», lo que está queriendo decir $\mathrm{A}$ es que «x es sabroso para mí», mientras que $\mathrm{B}$ está queriendo decir que «x no es sabroso para mí», de manera que $\mathrm{A}$ y B no se están contradiciendo en realidad ${ }^{10}$, y por lo tanto no tiene sentido ni siquiera pensar en que alguno de los dos haya cometido un error al expresar su criterio de gusto ${ }^{11}$. Cuando expresamos algunos juicios de valor no los formulamos, por así decir, de manera completa; pero implícitamente estamos haciendo referencia a nuestros propios criterios - en este caso en materia de gusto-.

Lo que se infiere de todo lo anterior es que, en estos ámbitos, se habla de dos tipos diferentes desacuerdos:

- desacuerdos que involucran error,

- desacuerdos que no involucran error («desacuerdos intachables»).

Además, hemos visto, tal y como afirma el relativismo indexical, que hay situaciones «intachables», esto es, situaciones en las que en realidad no hay un desacuerdo porque no se apela a un criterio compartido.

Pero dentro de los desacuerdos que involucran error creo que hay diferentes grados de error que tal vez convendría destacar, para ello usaré algunos ejemplos:

3. A afirma que Colón nació en Génova, mientras que B afirma que nació en Barcelona.

4. A afirma que Leo Messi nació en Rosario, mientras que B afirma que nació en Córdoba.

5. A afirma que $2+2$ suman 4 , mientras que $B$ dice que suman 5 .

El desacuerdo (a) es un desacuerdo en el que, necesariamente, una de las dos partes está cometiendo un error, y esto puede decirse a pesar de que, por lo menos hasta el momento, es imposible saber cuál de las dos partes está en error ${ }^{12}$.

8 IACONA, 2008: 288.

9 IACONA, 2008: 288.

10 IACONA, 2008: 288.

11 También en este caso es posible que puedan darse errores: si pruebo un pesto que me han dicho que ha sido hecho con la albahaca de Pra, pero en realidad la albahaca no es de Pra, el juicio según el cual «el mejor pesto es el que contiene albahaca de Pra» contendrá algún error. Pero este no es el sentido de error que aquí nos interesa.

12 Cabría la posibilidad de que Colón no hubiera nacido en Génova ni en Barcelona. Pero imaginemos, por hipótesis, que Génova y Barcelona son las únicas posibilidades. 
El desacuerdo (b) también es un desacuerdo en el que necesariamente una de las dos partes está cometiendo un error, pero, a diferencia del desacuerdo (a), aquí sí que es posible saber con relativa facilidad cuál de las dos partes ha cometido el error y, por tanto, resolver el conflicto.

En el desacuerdo (c) una de las dos partes ha cometido un error, el agente B, y su error es tan clamoroso que nadie duda de que él es la parte que ha cometido el error.

En los tres tipos de desacuerdo una de las partes ha cometido un error: pero mientras en (a) es imposible de descubrir, en (b) basta — por ejemplo- con consultar Internet y en (c) el agente B sencillamente no ha entendido nada de las reglas de la aritmética. Los tres son desacuerdos que involucran un error, pero en este caso se trata de errores gradualmente graves, y esto debería ser tenido en cuenta a la hora de hacer una taxonomía de los desacuerdos que involucran error ${ }^{13}$.

\section{CONCEPCIONES DEL DERECHO Y DESACUERDOS JURÍDICOS}

A continuación voy a enumerar dos concepciones hipotéticas en el derecho según cuál sea su posición respecto de los desacuerdos jurídicos. Pero antes de ello voy a reconstruir mínimamente en qué consiste el debate acerca de los desacuerdos jurídicos en el seno de la teoría del derecho contemporánea.

\subsection{El segundo acto del debate HART-DWORKIN y los desacuerdos interpretativos}

La cuestión de los desacuerdos jurídicos ha ocupado buena parte del debate iusfilosófico en las últimas décadas, al menos en el mundo angloamericano. Sin embargo, lo que está en discusión en aquel debate se encontraba presente ya, aunque en otros términos, en la obra de algunos autores «continentales» de mitad de siglo XX: cuando Ross constataba la existencia de una ideología dominante de las fuentes, cuando KELSEN hablaba del marco interpretativo o cuando el realismo jurídico genovés, con TARELLO a la cabeza, ponía el acento en el escepticismo interpretativo, se estaba hablando, implícita o explícitamente, de los desacuerdos jurídicos.

Sin embargo, la trama que yo usaré aquí para presentar el problema de los desacuerdos jurídicos se apoya en el debate HART-DwORKIN.

Según DwORKIN, el positivismo jurídico — hartiano— no puede dar cuenta de las disputas que los jueces tienen acerca de qué cuenta como un «ground of law», esto es, una base de derecho. DwORKIN distingue entre «propositions of law» (proposiciones de derecho) y «grounds of law» (bases de derecho) ${ }^{14}$. Una proposición de dere-

13 Por ejemplo, yo estaría dispuesto a afirmar, aunque no lo desarrollaré aquí, que llamar «desacuerdo»al desacuerdo (c) carece de sentido; yo me inclinaría, por oposición a las situaciones de «faultless» o situaciones «intachables», por denominarlo situaciones «fault» o situaciones «viciadas o con tacha», esto es, situaciones en que el error es tan palmario que el inicio de la discrepancia deviene prácticamente ridículo. A los desacuerdos (b) los llamaría desacuerdos «actualmente resolubles» y a los desacuerdos (a) los llamaría desacuerdos «actualmente irresolubles».

14 DwORKIN, 1986: 4 y ss. 
cho es un enunciado acerca del contenido del derecho de un cierto sistema jurídico. Por ejemplo: «está prohibido circular a una velocidad superior a $50 \mathrm{~km} / \mathrm{h}$ en Miguel Ángel de Quevedo». Las proposiciones de derecho pueden ser verdaderas o falsas. Serán verdaderas en virtud de las bases de derecho. En Ciudad de México, la anterior proposición será verdadera - simplificando las cosas- si una mayoría de miembros del órgano legislativo correspondiente del D. F. la ha aprobado. Las leyes aprobadas por el órgano legislativo hacen que la proposición de derecho sea verdadera y, a la vez, cuentan como base de derecho en el sistema jurídico municipal de Ciudad de México.

En lenguaje no dworkiniano, por tanto, una base de derecho tiene que ver con los criterios de validez jurídica.

Con arreglo a esta distinción, DwORKIN afirma que pueden darse dos tipos de desacuerdos: cuando dos o más jueces desacuerden acerca de si han tenido lugar o no las bases de derecho (esto es: ¿ha sido aprobada por la mayoría requerida la ley X?), tendrán un desacuerdo empírico; cuando dos o más jueces desacuerdan acerca de qué cuenta y cómo identificamos las bases de derecho (esto es: ¿qué hace que una base de derecho sea una base de derecho: basta, por ejemplo, con que haya seguido un determinado procedimiento legislativo o es necesario también algún requisito que tenga que ver con su contenido?), tendrán un desacuerdo teórico.

Los desacuerdos empíricos son más bien infrecuentes. En cambio, los desacuerdos teóricos, según DwORKIN, son abundantes.

El positivismo jurídico, en su reconstrucción dworkiniana, está comprometido con dos tesis: en primer lugar, sostiene que las bases de derecho, los criterios de validez jurídica, están determinados por consenso entre los officials, de forma que si acuerdan que los hechos del tipo $b$ son bases del derecho en su sistema, entonces los hechos del tipo $b$ son bases del derecho; en segundo lugar, sostiene que sólo determinados tipos de hechos pueden ser bases del derecho: los denominados hechos históricos claros (plain historical facts) ${ }^{15}$.

Si esto es así, en el marco conceptual del positivismo jurídico no hay espacio para los desacuerdos teóricos: si los participantes deben estar de acuerdo acerca de qué cuenta como una base de derecho, se sigue que no pueden estar en desacuerdo. Todo desacuerdo, en el marco conceptual del positivismo jurídico, versará sobre hechos: los hechos que hacen que una base de derecho sea una base de derecho. Sin embargo, los jueces a veces parecen no discrepar acerca de cuestiones empíricas, sino sobre qué quiere decir una base de derecho, sobre qué hace que un criterio de validez jurídica sea un criterio de validez jurídica.

DwORKIN aduce que una buena teoría del derecho debe poder dar cuenta de este fenómeno.

Hasta aquí el relato de la discusión que ha dominado los últimos años el debate académico iusfilosófico angloamericano. Ahora, siguiendo algunas ideas de G. B. RAT$\mathrm{TI}^{16}$, haré algunas precisiones.

15 SHAPIRO, 2007: 37.

16 RatTI, 2008. 
Cuando se habla de desacuerdos acerca de las «bases del derecho», para usar el léxico que DwORKIN puso de moda, no queda muy claro si se está hablando de desacuerdos acerca de cuáles son las fuentes del derecho o bien de desacuerdos acerca de cuál es el contenido de significado de las fuentes.

El primer tipo de desacuerdo, pese a que se da, es más bien poco común, y ni siquiera los realistas jurídicos de diverso pelaje le dan mucha importancia ${ }^{17}$. Parece ser el segundo tipo de desacuerdo el que resulta relevante y el que, como veremos, tal vez pueda contribuir a dirimir qué concepción del derecho resulta más plausible.

Los desacuerdos acerca de cuál es el significado de las fuentes pueden ser denominados «desacuerdos interpretativos», ya que la interpretación jurídica es la actividad en que el intérprete —el juez o el official de turno- atribuye, o descubre, según cuál sea la teoría de la interpretación que se suscriba, un determinado significado a la fuente ${ }^{18}$.

En lo que sigue, asumiré que los desacuerdos interpretativos se dan en el derecho y que al menos algunos de ellos pueden ser reconstruidos como DI. Si esto es así, ¿qué diversas maneras existen de dar cuenta de los DI en el derecho?

\subsection{Concepciones del derecho}

En esta sección presentaré algunas grandes concepciones del derecho. No tomaré como referente a ningún autor en concreto para llevar a cabo esta reconstrucciones que, por lo demás, serán bastante sucintas. Lo anterior quiere decir que mi interés no descansa tanto en que algunos autores puedan suscribir por completo alguna de las concepciones que yo presentaré, sino que más bien me interesa delimitar conceptualmente cada una de las concepciones sobre la base de qué dirían acerca de los desacuerdos jurídicos ${ }^{19}$.

Irenismo. Para esta concepción, el derecho es armónico y todo caso jurídico tiene una y sólo una respuesta jurídica correcta susceptible de ser conocida mediante el raciocinio.

El irenismo sostiene que el derecho está globalmente determinado, puesto que todo caso tiene una única respuesta jurídica correcta, y a la vez es armónico, esto es, es posible ordenar o jerarquizar todos los valores o bienes jurídicos de forma tal que nunca haya pérdida para ninguna de las partes. Pese a las apariencias, determinación global y armonía no son la misma propiedad; existe espacio conceptual para pensar que el derecho tiene siempre una única respuesta jurídica correcta para todo caso y

17 A. Ross sostenía, como es sabido, que cuáles son las fuentes del derecho no es algo que esté determinado por ningún criterio objetivo o racional, sino que depende de las ideologías — básicamente- de los jueces, pero sin embargo hay una ideología dominante de las fuentes, esto es, un núcleo de acuerdo generalizado alrededor de cuáles son las fuentes válidas de derecho, Ross, 1958. B. LEITER, por su lado, recalca que nadie pone en duda que la Biblia o el suplemento de economía de un periódico no son fuentes válidas de derecho, LEITER, 2009: 1217.

${ }^{18}$ Nótese que, de la manera en que yo lo estoy presentando, el desacuerdo interpretativo presupone un acuerdo acerca de las fuentes.

19 Lo que no será óbice, obviamente, para que el lector familiarizado con esta literatura asocie de manera prácticamente inmediata a determinados autores con las concepciones en concreto. 
sin embargo que ello ocurre a costa de algún tipo de pérdida en materia de valores o bienes jurídicos.

Para esta concepción los desacuerdos jurídicos interpretativos serán solamente aparentes. Siempre habrá alguna de las partes que habrá cometido algún tipo de error. Se trataría entonces de desenmascarar ese error y por lo tanto quedarse con la respuesta correcta.

Es importante destacar que para esta concepción la respuesta unívoca no es evidente, en el sentido de que admite la presencia de desacuerdos aparentes. Digo esto porque de lo contrario se trataría de una posición realmente naîf que ignoraría los disensos que, en el seno de la práctica del derecho, se producen con bastante frecuencia.

Me parece que el irenismo, para ser tomado como una posición relevante, debe admitir que se producen tales disensos para, a continuación, poder decir que son meramente aparentes.

Ha habido diversas estrategias que afirman, aunque no con estas palabras, un derecho irenista y que precisamente intentan mostrar que los desacuerdos son sólo aparentes. Las que han tenido más recorrido en nuestra cultura jurídica son sobre todo tres: el especificacionismo, la teoría del derecho como integridad y la ponderación.

El especificacionismo afirma que se puede tener una lista exhaustiva de todas las propiedades relevantes del caso que conforman el antecedente de la norma jurídica. Una vez especificada esa lista, los casos de desacuerdo devendrán meramente aparentes, porque habremos refinado el supuesto de hecho y ya sólo una de las opciones será realmente aplicable al caso. En otras palabras: el desacuerdo será superficial y una vez hayamos profundizado en el caso concreto nos daremos cuenta de que en realidad siempre hubo una sola respuesta correcta ${ }^{20}$. El error, para esta estrategia, se encontraría en la falta de especificación del antecedente de la norma que desciende del valor o bien jurídico a proteger.

La teoría del derecho como integridad, ésta sí atribuible a un autor concreto, R. DwORKIN, afirma que la respuesta correcta, para todo caso jurídico, será aquella que mejor encaje y más coherente sea con los valores que explican y justifican la existencia de un sistema jurídico concreto. En cualquier caso, el error en este caso residiría en no haber entendido el alcance de un determinado bien jurídico y por lo tanto no haber identificado la respuesta correcta ${ }^{21}$.

La teoría de la ponderación ha sido entendida de formas diversas. La que interesa en esta sede consiste en que cuando hay un conflicto entre dos principios jurídicos ${ }^{22}$,

${ }^{20}$ J. J. MORESO, sobre algunas ideas de R. M. HARE, ha ensayado esta estrategia mucho más en profundidad de lo que yo lo he hecho en el texto, vid. MORESO, 2002: 82 y ss.

${ }^{21}$ Es el argumento que, a grandes rasgos, también usó DwORKIN para replicar la idea berliniana del conflicto entre libertad e igualdad: según DwORKIN, quien piense que libertad e igualdad son dos valores in compatibles es que no habrá entendido el verdadero alcance de ambos valores, DwORKIN, 2001. Por lo demás, es sabido que la teoría de la integridad dworkiniana es realmente intrincada, por lo que difícilmente se podrá dar cuenta aquí de manera sintética de sus ideas. De todas formas, donde ha defendido de forma más explícita la idea de la única respuesta correcta es en DwORKIN, 1985: 119-145 y en DwORKIN, 1986.

${ }^{22}$ Nótese que la ponderación se aplica sólo en caso de conflictos entre principios, no entre reglas. Para este último supuesto, se aplica normalmente los criterios de resolución de antinomias (lex specialis, lex superior, 
normalmente de rango constitucional, estos son pesados, esto es, a cada uno de ellos es atribuido un determinado peso en un caso concreto. De esta manera al final sólo un principio, aquel que tenga mayor peso, es aplicable al caso concreto. En este caso el error consistiría en llegar a la conclusión de que hay dos principios empatados, a saber, de igual peso; para los partidarios de la ponderación, una situación de empate indica alguna equivocación en el momento de pesar los ítems ${ }^{23}$.

En cualquier caso, estas tres estrategias, presentadas aquí de manera muy somera por razones de espacio, están destinadas a un mismo objetivo: encontrar o descubrir la respuesta jurídicamente correcta y que ello no suponga pérdida alguna. Así pues, estas tres estrategias presuponen que el derecho puede ser armónico.

Las analogías estructurales entre realismo semántico e irenismo resultan bastante fáciles de advertir: para ambas todo desacuerdo presupone algún tipo de error por parte de alguna de las partes.

El irenismo, así pues, no provee una explicación para el fenómeno de los DI en el derecho. $\mathrm{O}$, mejor dicho, en la explicación irenista no ha lugar para los DI en el derecho.

Escepticismo. Para esta concepción, el derecho es conflictualista y para casi todo caso jurídico hay una pluralidad de respuestas jurídicas (y no hay forma de conocer mediante el raciocinio cuál de ellas es la respuesta jurídica correcta).

Para esta posición a toda o a casi toda disposición normativa se le puede atribuir una pluralidad de significados ${ }^{24}$, de forma tal que el derecho estaría globalmente indeterminado, y la respuesta a los casos jurídicos dependería del intérprete del derecho que, sobre la base de las tesis dogmáticas que le han influido y de sus preferencias ideológicas, optaría por (elegiría) una de las respuestas.

Por otro lado, la elección del intérprete significará que aquellos que optaban por otras respuestas perderán. En este sentido, el derecho, para los escépticos, no es armónico; los escépticos preferirán hablar de conflictos de intereses más que de desacuerdos aparentes. Contrariamente a lo que afirman los irenistas, el derecho no satisface todos los intereses de sus participantes; el derecho es el mundo del conflicto, no de la armonía.

También en este caso el paralelismo con el relativismo indexical es muy intuitivo: cuando el intérprete de turno, el juez A, por poner el ejemplo más visible de intérprete del derecho, elige la respuesta $\mathrm{X}$ y el juez $\mathrm{B}$, suponiendo que se trate de un órgano colegiado, elige la opción $\mathrm{Y}$, respecto de la disposición normativa $\mathrm{D}$, en realidad no están teniendo un desacuerdo: lo que están queriendo decir realmente es que, según sus criterios de interpretación, para $\mathrm{A}$ la respuesta es $\mathrm{X}$ y, según sus criterios de interpretación, para B la respuesta es Y. Así que cada uno de los participantes en la disputa está apelando a criterios diferentes y no está contradiciendo la respuesta que ha elegido el otro.

\footnotetext{
lex posterior), pero el resultado es el mismo que en el caso de la aplicación de la ponderación para los principios: una única respuesta jurídica correcta para el caso concreto.

${ }^{23}$ El autor que más ha insistido en esta cuestión es R. ALEXY, quien incluso ha formulado la cuestión de la ponderación en términos aritmético, vid. por ejemplo ALEXY, 1986: 81-98.

${ }^{24}$ Guastini, 2009.
} 
Y mientras que los irenistas decían que los desacuerdos en el derecho eran aparentes, los escépticos afirman que los acuerdos que se dan en el derecho son tendencialmente aparentes. El derecho es sobre todo conflicto de intereses y desacuerdo; esto a veces puede quedar oculto por dos razones: (i) casos en que hay relaciones de cooperación y en que hay convergencia de intereses y (ii) casos en que hay una respuesta unívoca por parte de un tribunal a un caso jurídico concreto. En casos de (i), tales como los contratos o el matrimonio, el acuerdo se produce simplemente porque hay normas jurídicas que prevén sanciones en caso de violar los términos de esa relación de cooperación, es decir, en otras palabras: se presupone también ahí un caso de conflicto potencial. Que haya acuerdo o no en cualquier caso será una cuestión contingente. En casos de (ii), ocurrirá sencillamente que el «soberano» (el juez que tenga mayor fuerza o que consiga mayor aquiescencia en el órgano colegiado de turno) impondrá su decisión y las respuestas alternativas quedarán solapadas porque el tribunal en realidad está obligado a tomar una única decisión, dando así la impresión de que hay un acuerdo entre los miembros del órgano colegiado ${ }^{25}$.

En ambos casos vemos que la estructura del derecho responde a la de un soberano (personificado en el caso concreto en un juez determinado) que imparte órdenes bajo amenaza de sanción.

El derecho, por lo tanto, estaría globalmente indeterminado y la respuesta a cada caso jurídico siempre sería relativa a las preferencias ideológicas e influencias dogmáticas del soberano (el juez del caso particular) de turno.

Así pues, para el escepticismo en el derecho no habría casos de DI; sólo habría casos de conflictos de intereses o de acuerdo aparente. Los casos jurídicos podrían ser reconstruidos como casos sencillamente intachables, puesto que el escepticismo sostiene una posición anti-cognitivista en materia práctica, por lo que no tendría sentido predicar error de la elección de preferencias o intereses ${ }^{26}$. En otras palabras, los participantes en el derecho nunca cometerían error cognitivo alguno, pero tampoco estarían involucrados en desacuerdos genuinos, puesto que cada uno apelaría a sus propios criterios de interpretación. Es por ello que en el imaginario escéptico, como en el imaginario relativista, no ha lugar para los DI.

\section{LAS LIMITACIONES DE LOS BINOMIOS REALISMO/IRENISMO Y RELATIVISMO/ESCEPTICISMO}

De las presentaciones de las concepciones semánticas y del derecho presentadas hasta ahora parece seguirse que ni en el derecho ni en materia moral, estética o de gusto hay DI. Sin embargo, existen buenas razones para pensar que por lo menos respecto de algunas de las situaciones que se dan estos ámbitos, tiene sentido decir que son situaciones de DI. Lo que haré a continuación es señalar las limitaciones de cada una de estas concepciones.

${ }^{25} \mathrm{El}$ acuerdo aparente será más fácil de ocultar en aquellos sistemas jurídicos que no prevean instituciones como el voto particular o la «dissenting opinion».

${ }_{26}$ Esto comprometería conceptualmente al escepticismo con el emotivismo o con alguna otra forma de antirrealismo en metaética. 
Realismo/irenismo. Recordemos una vez más la tesis isomórfica del realismo y del irenismo. Tanto el uno como el otro sostienen que en los casos de (aparente) desacuerdo una de las partes habrá cometido un error.

Sin embargo, existen algunas disputas respecto de las cuales nos inclinamos por decir que se trata de discrepancias que no involucran error; y esto ocurre tanto en los ámbitos con los que el realismo lidia (gusto, estética, moral; recuérdense los ejemplos anteriormente mencionados) ${ }^{27}$, como con algunos de los desacuerdos jurídicos que encontramos en el derecho.

Esta posición no parece muy fundada porque, en primer lugar, descarta sin más matices que existan DI y, en segundo lugar, no da una explicación al hecho de que con según qué desacuerdos nos inclinamos por decir que son DI y con según qué desacuerdos somos más bien partidarios de decir que no lo son. El realismo no cree necesario responder a ninguna de estas dos intuiciones ${ }^{28}$.

De una forma parecida, el irenismo no puede dar cuenta de la idea según la cual no todos los desacuerdos jurídicos son aparentes y el derecho no siempre es armónico, en el sentido de que no siempre satisface las pretensiones de todos sus participantes. En otras palabras: el irenismo no puede dar cuenta de aquellos casos en que, por así decir, una de las partes pierde algo considerado valioso desde un punto de vista jurídico $-\mathrm{y}$ no sólo por parte de quien está directamente involucrado en el caso, sino también por parte de la comunidad jurídica en general-.

Por otro lado, el irenismo estaría dispuesto a decir que incluso en aquellos casos en los que encontremos disposiciones normativas que contengan términos vagos o ambiguos, el derecho está determinado, pues hay criterios objetivos (a saber, no dependientes de las opiniones de los jueces) que nos permitirán saber cuál es el significado unívoco de la disposición normativa al menos para el caso concreto. A este respecto,

${ }^{27}$ El realismo, por tanto, «do not offer any consolation to those who found it initially plausible that there could be faultless disagreements» (KöLBEL, 2003: 58), en los ámbitos señalados en el texto.

28 Uso aquí el término «intuición» porque es el que es usado, y aceptado, habitualmente en esta literatura. Sin embargo, me parece que alguna señal empírica de la existencia de DI puede ser aducida: me refiero a la perpetuidad de algunos desacuerdos. Que un desacuerdo no se resuelva nunca no prueba, en sentido estricto, nada; pero sí que puede ser considerado un síntoma de que tal vez la mejor manera de tratarlo sería como un DI. Es posible objetar que no todo el mundo tiene por qué compartir esta intuición, y que, por ejemplo, alguien puede tener otra «intuición»: la de que en realidad siempre hay una respuesta correcta y que una de las partes ha cometido un error. Al respecto, querría hacer dos observaciones: la primera de ellas consiste en que para desacreditar una «intuición» no basta con poner encima de la mesa otra «intuición» que contradiga a la primera, ya que la última adolecerá de los mismos problemas que la primera, a saber, se tratará precisamente de una «intuición», no de un argumento. Así que para desacreditar la intuición de que existen desacuerdos intachables en algunos ámbitos no basta con proponer una intuición alternativa y contraria; no compartir una intuición no es un argumento en contra de esa intuición. A modo de segunda observación, considero que es más razonable sostener la intuición de que existen desacuerdos intachables por la razón aportada en esta misma nota: la durabilidad y persistencia de algunos desacuerdos no prueba nada en sentido estricto pero sí que ofrece buenas razones para pensar que quizás el mejor modo de tratar a algunas situaciones es como instancias de desacuerdos intachables. Por otro lado, aunque es cierto que en la filosofía analítica el término «intuición» no goza de buena prensa, lo cierto es que a menos que uno presuponga una muy barroca ontología de los conceptos lo que hacemos todos cuando llevamos a cabo análisis conceptual es sistematizar y ordenar nuestras intuiciones; que no lo presentemos así casi nunca, y que tendamos a omitir el término «intuición», porque consideramos que empobrece y debilita nuestros argumentos es una cuestión a estudiar, desde luego, pero no enmienda la constatación de que muchos de nuestros argumentos y razonamientos parten de intuiciones que, confesadas o no, asumimos como premisas y cuya elección no siempre es susceptible de ser justificada racionalmente. 
valen las consideraciones hartianas respecto de los supuestos en los que el juez actúa discrecionalmente. De haber, como dicen los irenistas, criterios independientes de las opiniones de los jueces, no existirían casos en los que los jueces interpretan diversamente un mismo término, a saber, casos de desacuerdos interpretativos. El irenista podría entonces sacar a colación su leitmotiv: uno de los jueces estará necesariamente en un error. Pero parece difícil sostener que están en un error cuando la disputa es permanente y nunca queda zanjada ${ }^{29}$.

$\mathrm{Al}$ igual que el realismo, el irenismo tampoco provee criterios que nos permitan diferenciar aquellos casos jurídicos respecto de los cuales estamos inclinados por decir que se trata de DI de aquellos respecto de los cuales nos inclinamos por afirmar que no se trata de $\mathrm{DI}^{30}$.

Relativismo/escepticismo. El relativismo indexical sostiene que los casos de DI no son en realidad casos de desacuerdos, sino únicamente situaciones intachables, ya que ninguna de las partes ha cometido error alguno. Las partes hacen referencia a estándares diferentes, su elección es relativa a tales y en consecuencia no están en contradicción la una con la otra; ni siquiera tiene sentido preguntarse acerca de si una de ellas está en error.

El escepticismo afirma que la respuesta para cada caso jurídico será relativa a los estándares o criterios de interpretación de cada intérprete del derecho. Tampoco para el escepticismo, entonces, habría contradicción entre las respuestas que los diversos intérpretes darían a la interpretación de una disposición normativa a un mismo supuesto de hecho, ya que cada intérprete basaría su respuesta en sus propios criterios de interpretación ${ }^{31}$.

29 También en las ciencias duras, en las que habitualmente se considera que hay respuestas correctas más allá de las opiniones de sus participantes, existen desacuerdos. Sin embargo, los desacuerdos en ciencias duras no acostumbran a ser perpetuos: más tarde o más pronto - ahora estoy simplificando algo la cuestión - se suele detectar el fallo o error cometido por una de las teorías en competición. Los desacuerdos acerca de los significados de las palabras en materias prácticas —esto es, en ámbitos en los cuales suele haber intereses en juego-, y que además versan sobre términos vagos, son mucho más difíciles de zanjar y esto sería una señal de que tal vez conviene más tratarlos como DI.

${ }_{30}$ «[Realism] does nothing to account for the difference between those topics on which we are inclined to say that faultless disagreement is possible and those where we are not» (KÖLBEL, 2003: 58).

${ }_{31}$ Podríamos imaginar una versión algo diferente de la concepción escéptica en la cual el significado no dependiera del juez singular que interpreta la disposición normativa, sino de la interpretación vigente (respecto de esa disposición normativa) en la comunidad jurídica de referencia. Ante una concepción así, cabría hacer algunas consideraciones: (i) que no dependa de un solo sujeto, sino que dependa de una pluralidad de sujetos (i. e. de la comunidad jurídica) no cambia, en sentido estricto, el estatuto lógico de la actividad interpretativa: pasaría de ser subjetivo a inter-subjetivo, pero en ningún caso podría predicarse objetividad respecto de la actividad interpretativa; (ii) debido a lo anterior, para el escepticismo, cuando un juez no toma la interpretación vigente, podría decirse que se ha desmarcado de la opinión mayoritaria de su cultura jurídica, pero no podría decirse que su interpretación es incorrecta (puesto que, en última instancia, la interpretación responde a las preferencias ideológicas y a las asunciones dogmáticas del juez, y respecto de esto, en el imaginario escéptico, no se puede predicar error ni incorrección). Sólo si por «inter-subjetivo» cabiera entender «objetivo» en el sentido de criterio independiente de las elecciones de un individuo —el juez del caso concreto- o de la suma de individuos - la comunidad jurídica - podría hablarse de interpretaciones correctas o incorrectas; si por «inter-subjetivo» no puede entenderse lo anterior, entonces sólo puede hablarse de interpretaciones conformes o disconformes con la interpretación vigente en una determinada comunidad jurídica. (Tal vez podría predicarse objetividad epistémica en el sentido de que es susceptible de conocimiento objetivo cuándo un juez ha llevado a cabo un interpretación conforme o disconforme a la interpretación vigente; pero, repito, esto no cambio el estatuto lógico de la actividad interpretativa). Quien estuviera dispuesto a sostener que el criterio 
El relativismo indexical resulta insatisfactorio o encuentra limitaciones en un caso como el siguiente, que es explicado en dos fases diferentes, relativo al gusto. En la primera fase, Riccardo y Paolo desacuerdan sobre si el Barolo es mejor que el Priorat. Éste sería un caso para el que parece que en principio el relativismo indexical tendría una explicación: cuando Riccardo está diciendo que el Barolo es mejor está diciendo que «para mi gusto, el Barolo es mejor vino», mientras que Paolo también está apelando sus propios gustos, es decir, está diciendo «para mi gusto, el Priorat es mejor vino». Por lo tanto, están apelando a estándares diferentes y no están en contradicción ${ }^{32}$. Pero qué ocurre si, en la segunda fase de la situación, llega un tercer amigo, Pierluigi, con una botella de la marca blanca de un supermercado cualquiera. Pierluigi, tras probar el Barolo, afirma «mi vino es mejor que el Barolo». A lo que Riccardo y Paolo responden, vehementemente, que no, que está en un error. Puede que la disputa entre Paolo y Riccardo acerca del Barolo y del Priorat sea «intachable» y que no constituya un desacuerdo, puesto que no están en contradicción. Pero hay situaciones similares en que apelar a los propios gustos parece ignorar algún tipo de error: parece que si Pierluigi apelara a sus propios gustos al discutir que el vino del supermercado es mejor que el Barolo, nos inclinaríamos por decir que ha cometido algún tipo de error.

En un caso como el de Riccardo, Paolo y Pierluigi, nos inclinamos por decir que se trata de un desacuerdo y que Pierluigi ha cometido un error de algún tipo. Pero para poder decir que Pierluigi está teniendo un verdadero desacuerdo con Paolo y Riccardo, y que no es sencillamente una situación intachable, tenemos que presuponer que en realidad hay algún tipo de estándar común entre los tres agentes que alcanza para afirmar que habrá casos en los que es posible decir que alguna de las partes estará cometiendo un error (Pierluigi respecto de Paolo y Riccardo) y casos en los que tal criterio no alcanzará y sencillamente se tratará de situaciones intachables (la disputa entre Paolo y Riccardo).

El escepticismo también encontraría dificultades a la hora de dar cuenta de algunos casos en el derecho que no responden a la estructura del conflicto de intereses en el cual el soberano de turno impone su elección. Son conocidos los argumentos que HART adujo contra la concepción austiniana y benthamiana, de impronta imperativista, en los primeros capítulos de The Concept of Law $^{33}$. La idea general es siempre la misma: la intervención del derecho no es siempre coactiva; lo que significa que los

inter-subjetivo permite cribar interpretaciones objetivamente correctas de interpretaciones objetivamente incorrectas se estaría situando fuera del marco conceptual del escepticismo; algo completamente legítimo, desde luego, pero entonces no podría sostenerse esta idea bajo el rótulo del escepticismo. Si, en cambio, el criterio inter-subjetivo sirve para cribar aquellas interpretaciones vigentes de las no vigentes, entonces seguiremos dentro del marco conceptual del escepticismo y, en consecuencia, afrontaremos las mismas dificultades de las que daré cuenta en los próximos párrafos, aunque, para ser honestos, tal vez las afrontaría en un grado menor: la indeterminación del derecho no sería global, puesto que las interpretaciones vigentes nos permitirían algún grado de predictibilidad, pero en todo caso nada impediría al intérprete de turno el poder desmarcarse de la interpretación vigente y por lo tanto volver a elevar el grado de indeterminación. Para esta visión, digamos, inter-subjetiva del escepticismo, vid. FERRER BELTRÁN, 2012.

32 En este sentido, para que se diera una contradicción Riccardo tendría que decir, por ejemplo, que «de acuerdo con los gustos de Paolo, el Barolo es mejor», mientras que Paolo tendría que seguir diciendo «de acuerdo con mis gustos, el Priorat es mejor». Pero esta sería una situación más bien anómala, a no ser que alguien esté dispuesto a sostener una tesis cognitivista muy fuerte en materia de acceso a los estados mentales ajenos.

33 Vid. HART, 1961: 26 y ss. 
participantes en el derecho no siempre están enzarzados en conflictos de intereses cuya resolución, según el imaginario imperativista, siempre pasa por una orden del «soberano» (en el caso particular o en general) respaldada por la amenaza de una sanción: hay figuras jurídicas en las que prima la cooperación y el resultado satisface los intereses de todos sus participantes. Para ilustrar esta idea, transcribo un pasaje bastante conocido de HART en el que se ve clara la distinción entre diferentes tipos de normas jurídicas:

«Surely not all laws order people to do or not to do things. Is it not misleading so to classify laws which confer powers on private individuals to make wills, contracts, or marriages, and laws which give powers to officials, e.g. to a judge to try cases, to a minister to make rules, or a county council to make by-laws? Surely not all laws are enacted nor are they all the expression of someone's desire like the general orders of our model. This seems untrue of custom which has a genuine though modest place in most legal systems. Surely laws, even when they are statutes deliberately made, need not be orders given only to others. Do not statutes often bind the legislators themselves? Finally, must enacted laws to be laws really express any legislator's actual desires, intentions, or wishes? Would an enactment duly passed not be law if (as must be the case with many a section of an English Finance Act) those who voted for it did not know what it meant?» ${ }^{34}$.

Algunos negocios jurídicos, como el testamento o el contrato, no responden a la estructura de los participantes con conflictos de intereses. Para el escepticismo, lo jurídico es sinónimo de conflicto y de desacuerdo. Sin embargo, figuras como las anteriormente mencionadas parecen desmentir la idea escéptica.

El escéptico tal vez podría responder que si bien es cierto que la firma de un contrato, por ejemplo, no responde a la estructura del conflicto de intereses (y, por tanto, del desacuerdo entre los participantes), la interpretación del mismo, o la satisfacción de algunas de sus cláusulas, sí que puede generar desacuerdos. Y es ahí donde el derecho interviene coactivamente y donde probablemente sea más dudoso decir que todos los intereses de los participantes quedan satisfechos (si hay una controversia entre los participantes acerca de cómo debe ser interpretada una determinada cláusula del contrato, cuando el juez decida cómo debe ser interpretada una de las partes probablemente se verá perjudicada). Pero lo cierto es que no todos los contratos generan dudas acerca de su cumplimiento o de su interpretación y, por tanto, no requieren la intervención del «soberano» para resolverlos. Esto es, ni mucho menos — tómese esta afirmación como una tesis de sociología jurídica folk - todos los contratos terminan en los juzgados; las más de las veces los firmantes de un contrato lo cumplen y no hay controversia acerca de su interpretación porque el cumplirlo es precisamente beneficioso para ambos ${ }^{35}$.

Así pues, lo que pone de manifiesto el escepticismo es que el derecho estaría plagado de casos intachables pero no de DI. Podría decirse, por un lado, que el escepticismo acierta allí donde el irenismo fallaba, i. e. el derecho no es completamente armónico, y,

34 HART, 1961: 26.

35 Un escéptico tal vez podría añadir que el derecho existiría porque siempre cabría la posibilidad de que apareciera la figura del «free rider». Pero el «free rider» sólo tiene sentido porque sabe que, las más de las veces, los contratos se cumplen; si las más de las veces no se cumpliera, como parece afirmar el escéptico, entonces el «free rider» probablemente no surgiría, porque su razón de ser descansa en el provecho que saca de aquellos que efectivamente siguen las reglas. En otras palabras, el «free rider» presupone que los contratos se cumplen las más de las veces; de otra manera, su existencia, la del «free rider», carecería de sentido. Debo esta idea a una sugerencia de I. LIFANTE. 
por otro lado, falla allí donde el irenismo acertaba, i. e. algunas relaciones jurídicas no responden a la estructura del conflicto de intereses y de las órdenes impartidas por el soberano bajo amenaza de sanción.

Volviendo a la cuestión semántica, tal y como dice IACONA, ninguna de las dos posibilidades, ni realismo ni relativismo indexical, parece compatible con la existencia de DI en los ámbitos de la moral, el gusto o la estética:

«Realism is taken to entail that all the disputes that qualify as good candidates for being faultless disagreements are disagreements. Since we are inclined to say that some disputes of that kind are faultless, the realist is left with the problem that some disagreements seem faultless. In the same way, indexical relativism is taken to entail that all the disputes that qualify as good candidates for being faultless disagreements are faultless. Since we are inclined to say that some disputes of that kind are disagreements, the indexical relativist is left with the problem that some faultless disputes seem disagreements» ${ }^{36}$.

Por otro lado, tampoco irenismo ni escepticismo resultan compatibles con la existencia de DI en el derecho por las razones aducidas anteriormente y que recordaré ahora a modo de síntesis.

Con arreglo al irenismo, el derecho es siempre armónico en el sentido del que he dado cuenta anteriormente. Esto quiere decir que no habrá situaciones en las cuales una parte y la otra están afirmando cosas contradictorias y una de ellas no esté en un error. Lo que es tanto como decir que no ha lugar para los DI en el derecho.

Por su parte, el escepticismo considera que la estructura básica del derecho es la del soberano que imparte órdenes y que las relaciones jurídicas son esencialmente relaciones de conflictos de intereses. Y en los casos de conflicto cada participante apela a sus propios estándares de interpretación, por lo que (i) no estamos en caso de contradicción (de desacuerdo genuino), porque se está hablando at cross purposes, y (ii) no se puede predicar error en materia de elección de criterios. Es decir, no ha lugar para DI en el caso del escepticismo.

\section{PLURALISMO DILEMÁTICO: UNA EXPLICACIÓN QUE DA CABIDA A LOS DI EN SEMÁNTICA Y EN EL DERECHO}

\subsection{Pluralismo dilemático: cuestiones conceptuales}

Lo que yo llamaré pluralismo dilemático, y que se basa en algunas consideraciones que I. BERLIN constató acerca de la moral ${ }^{37}$, afirma las siguientes tesis:

B. Los casos prácticos tienen, en algunas circunstancias, una pluralidad de respuestas, todas ellas correctas, debido a que los seres humanos no somos monistas acerca de las valores (estéticos, morales, jurídicos, etc.), sino que sostenemos una concepción pluralista acerca de aquello que consideramos como bueno.

C. En otras circunstancias, en cambio, el caso práctico tendrá una única respuesta, ya sea porque en realidad hay un único valor aplicable o porque, aun habiendo varios, pueden ser ordenados y jerarquizados sin pérdida.

\footnotetext{
36 IACONA, 2008: 290.

37 BERLin, 1990.
} 
D. Cuando un caso práctico tenga una pluralidad de respuestas, y éstas no puedan ser ordenadas jerárquicamente, nos encontraremos ante un caso de dilema trágico, esto es, un caso en el que se elija lo que se elija tendremos algún tipo de pérdida ${ }^{38}$. En otras palabras: en los casos de dilema trágico será imposible satisfacer todas los bienes (o intereses o valores, sean éstos morales, jurídicos o estéticos).

E. Las respuestas no podrán ser ordenadas jerárquicamente por dos motivos diversos: o bien porque carecemos de un criterio o estándar común de valoración entre los diferentes ítems, y se dirá entonces que nos encontramos ante casos de inconmensurabilidad; o bien porque, aun teniendo un criterio común que nos permita establecer tal valoración, ésta dará como resultado una situación de empate entre los ítems.

Es necesario recalcar que este modelo, con las características apenas mencionadas, es una alternativa tanto al realismo como al relativismo indexical, en materia semántica, como al irenismo y al escepticismo, en materia jurídica.

La estructura del conflicto o dilema trágico comparte rasgos con la de los DI. A saber, un disenso en el que una de las partes afirma $p$ y la otra afirma no- $p$ (o bien una afirma $p$ y la otra $q$, pero es imposible satisfacer $p$ y $q$ contemporáneamente) y ninguna de las dos ha cometido error, ya que ambas resultan ser aplicables al caso, es decir ambas son igualmente correctas.

Hay una diferencia que debe ser puesta aquí de relieve, para evitar equívocos, pero que no cambiará sustancialmente el modelo pluralista dilemático a los efectos de ser alternativa a los binomios ya enumerados. Los conflictos prácticos, según una conocida taxonomía de B. Williams, pueden ser al menos de tres tipos: intra-subjetivos, intersubjetivos e inter-culturales. Los del primer tipo se dan entre valores sostenidos por un mismo individuo. Los del segundo tipo se dan entre valores sostenidos por diferentes individuos. Mientras que, los del último tipo, se dan entre valores pertenecientes a diferentes épocas, culturas o lugares.

El caso de dilema trágico es tradicionalmente entendido, por ejemplo por parte de BERLIN, como un caso de conflicto intra-subjetivo, en el que un mismo sujeto es requerido para llevar a cabo, por ejemplo, dos obligaciones que, sin embargo, no puede llevar a cabo. El caso de los DI no responde normalmente a la estructura del conflicto intra-subjetivo, sino que, como en los ejemplos aportados anteriormente, hay una pluralidad de individuos: se trata de un caso de conflicto inter-subjetivo (o, incluso, aunque aquí no lo trataremos, podría ser reconstruido como un caso de conflicto inter-cultural). Así pues, estamos ante un escenario en que los dilemas trágicos son conflictos intra-subjetivos, mientras que los DI son conflictos inter-subjetivos. Se trata, efectivamente, de una diferencia existente entre una y otra situación. Pero se trata de una diferencia irrelevante, como he avanzado, para lo que yo pretendo sostener aquí, que es lo siguiente: tanto en casos de DI como en casos de dilema trágico no hay solución armónica posible, esto es, no hay solución que no suponga sacrificar (al menos parte de) alguno de los valores en liza ${ }^{39}$.

38 De hecho, aunque la jerarquización sea posible, esto no querrá decir que no podamos perder algo de valor.

39 Dependiendo de cuál sea la solución se puede estar sacrificando un valor en su totalidad o bien sólo en parte. Si esto fuera así, entonces podría decirse que el concepto de «sacrificio» es gradual. Pero esto no cambiaría las cosas: únicamente habría conflictos en los cuales se sacrifica más y conflictos en los que sacrifica 
Es necesario hacer una observación ulterior. Imaginemos una situación como la siguiente:

\section{A afirma Op \\ B afirma Oq}

En este caso, tanto p como q resultan ser igualmente aplicables al caso, pero no es posible llevar a cabo contemporáneamente ambas. Se trataría de un conflicto inter-subjetivo, puesto que hay dos participantes. En sentido estricto, un caso como este no se correspondería con un DI, ya que la respuesta correcta al caso no es ni Op ni Oq, sino Op\&Oq, por lo que tanto A como B estarían cometiendo sendos errores (a pesar de que sus respuestas son aplicables al caso). Nótese sin embargo que, en cualquier caso, no es posible llevar a cabo el estado de cosas que nos prescribe la respuesta correcta, esto es, p\&q, por lo que en cualquier caso nos encontramos ante un caso trágico.

Este caso es interesante porque hasta el momento tanto el realismo semántico como el irenismo siempre han sostenido que, necesariamente, si una de las partes estaba en un error entonces la otra no lo estaba. Pero este caso es diferente: ambas partes parecen estar cometiendo sendos errores. Tanto realismo como irenismo parecen asumir alguna versión del principio de bivalencia: si una de las partes afirma un enunciado falso o incorrecto entonces la otra afirma un enunciado verdadero o correcto. En el caso aquí planteado no parece darse la bivalencia, al menos entre los participantes en este desacuerdo; aquí, que un participante formule un enunciado falso, por ejemplo, $\mathrm{Op}$, no significa que el otro participante esté formulando un enunciado verdadero, es decir, que Oq sea verdadero. Pero dicho de este modo, parecería entonces que estamos ante un caso cuya mejor reconstrucción quedaría en manos de alguna versión (oportunamente modificada) de la teoría del error mackiana: los enunciados normativos para este caso resultan ser falsos - ambas partes están en un error-y de ello no se sigue que haya un enunciado verdadero. La teoría del error de MACKIE, en efecto, no parece en principio compatible con la ley de bivalencia. Pero una reconstrucción tal omitiría que en realidad sí hay un enunciado verdadero en nuestra situación, es decir Op\&Oq, que es el producto de la conjunción de ambos enunciados normativos. Así pues, si pasamos a observar la situación desde este punto de vista, los participantes en el desacuerdo no estaban equivocados: tanto Op como Oq eran aplicables al caso y eran correctos, lo que ocurre es que, por así decir, no lo eran por separado, sino en conjunto.

Por otro lado, el punto concomitante entre conflicto trágico y DI consiste en que cuando digo que una situación del tipo anterior no involucra ningún error lo que quiero decir es que la conjunción de las opciones de ambos participantes no involucra ningún error. La respuesta correcta al caso, asumir que ambas opciones son igualmente correctas, aunque incompatibles, no denota ningún error. S. HAMPSHIRE lo ha expresado así: «Neither in social order, nor in the experience of an individual, is a state of conflict of a vice, or a defect, or a malfunctioning» ${ }^{40}$. El conflicto trágico se da a nivel intrasubjetivo, inter-subjetivo o, como dice HAMPSHIRE, incluso a nivel social; $y$, cuando ello ocurra, nada nos debe hacer creer que hay algo defectuoso en nuestro pensamiento, ni

menos, pero en ambos casos se trataría de situaciones en las que no podemos satisfacer completamente los valores relevantes.

40 HAMPSHIRE, 2000: 33. 
tampoco debemos creer que porque estemos en conflicto, con nosotros mismos, o en desacuerdo, con un tercero, hemos cometido - o el otro ha cometido- algún tipo de error. El desacuerdo, el conflicto, no es un indicio siempre y de manera necesaria, ni mucho menos, de que algo hemos -o han - hecho mal, sobre todo cuando hablamos del ámbito práctico. Es en este sentido en que es relevante el hecho de que FI y conflictos trágicos compartan rasgos.

\subsection{Virtudes del pluralismo dilemático ${ }^{41}$}

En primer lugar, el pluralismo dilemático no afirma que todo caso sea susceptible de conflicto trágico o de DI. Sólo en determinadas circunstancias determinados valores (que pueden ser sostenidos por una misma persona o por dos o más agentes) entrarán en conflicto. Esto es, la multiplicidad de valores no será conflictiva en todos y cada unos de los casos: en determinados casos las circunstancias permitirán una armonía o jerarquización sin pérdida de los valores en juego. En el pluralismo dilemático, entonces, hay cabida para los casos de acuerdo o de falta de conflicto ${ }^{42}$.

En segundo lugar, el pluralismo dilemático puede superar las dificultades que plantea el realismo/irenismo, puesto que permite conservar la intuición según la cual existen algunos casos de DI en el derecho. ¿Y de qué casos se trata? Estamos hablando de los casos de conflicto o dilema trágico; esto es, casos en que o bien no hay un baremo común entre las dos opciones que nos permita elegir racionalmente entre ambas (casos de inconmensurabilidad) ${ }^{43}$, o bien hay un baremo pero los valores o conside-

41 Para BERLin, el pluralismo de valores (al que yo denomino pluralismo dilemático) se opone al monismo moral. El irenismo sería una forma sofisticada de monismo moral, pues tal y como dice G. CROWDER: «At its broadest, monism is the basis of the whole Western tradition of utopianism that reaches back to Plato, indeed of the mainstream of Western philosophy, the "perennial philosophy" (philosophia perennis) according to which all questions, including moral questions, have a single correct answer and all correct answers fit together like a jigsaw puzzle» (CROWDER, 2004: 115).

42 La tesis del dilema trágico entre valores puede ser entendida en el sentido de que el conflicto es (i) conceptual o (ii) contingente. La interpretación que he dado yo se corresponde con (ii). En este punto, BERLIN fue ambiguo, y en ocasiones parecía suscribir (i) (JAHANBEGLOO, 1990: 189) mientras que en otras ocasiones pareció suscribir (ii) (BERLIN, 1990, 13). Algunos de sus intérpretes parecen no haber sido capaces de deshacer esta ambigüedad; así, G. CROwDER afirma en un pasaje que «Obviously goods do not always conflict, but often they do, and then we have to make a choice» (CROWDER, 2004: 136), mientras que unas líneas más adelante dice que «For Berlin, political perfection is impossible, not merely in the sense that it is beset with practical difficulties, but "in principle", because so many fundamental buman goods are conceptually incompatible» (CROWDER, 2004: 137). $\mathrm{Si}$, como dice CROWDER en la última cita, la incompatibilidad entre los valores es de tipo conceptual, entonces siempre están en conflicto; pero entonces no puede ser verdad lo que afirma en la primera cita, esto es, que los valores no siempre entran en conflicto, aunque a menudo lo hagan. Mientras la segunda cita afirma el carácter 'necesario' del conflicto entre valores, la primera cita parece sugerir que el conflicto sería 'contingente'. En cualquier caso, y más allá de la exégesis del pensamiento berliniano, creo que lo más adecuado es pensar que los valores no están en conflicto ya en abstracto o de forma necesaria. Uno de los conflictos contemporáneos entre valores por antonomasia, el conflicto entre libertad e igualdad, surge casi siempre debido a unos circunstancias concretas (escasez de recursos, desigualdades sociales y económicas, etc.) de nuestro mundo real, circunstancias que sin embargo no tendrían por qué reproducirse en todos los mundos posibles. En la medida en que sea posible imaginar algún mundo posible cuyas circunstancias hagan que los valores no estén en conflicto, podemos pensar que el conflicto no será necesario, sino contingente (al respecto, véanse las afiladas reflexiones de BAYÓN, 1991: 407 y ss.).

43 Aunque no profundizaré aquí en la cuestión de la inconmensurabilidad, sí recordaré que hay dos maneras de entender qué quiere decir que dos ítems son inconmensurables: en primer lugar, se dice que dos 
raciones tienen el mismo peso (casos de empate). En estos casos, ninguna de las dos partes (o un solo agente, si se trata de un conflicto intra-subjetivo) habrá cometido ningún error y, por lo tanto, podremos decir que la hipótesis de reconstrucción que yo he denominado pluralismo dilemático consiente —es más, yo diría que constituye su tesis más original- la existencia de DI, entendidos tanto en el sentido intra-subjetivo como en el sentido inter-subjetivo.

En tercer lugar, el pluralismo dilemático también está en condiciones de superar la adversidad planteada al irenismo según la cual este último no proveía criterio alguno que permitiera distinguir entre aquellos casos en los que estamos inclinados por decir que se trata de DI y aquellos casos en que más bien somos partidarios de decir que no hay conflicto (esto es, en que, a pesar de las apariencias de conflicto, en realidad o bien sólo hay un valor aplicable al caso o bien se pueden armonizar de tal manera que no implique pérdida alguna). El criterio que provee el pluralismo dilemático, consiste en la ya mencionada distinción entre aquellos casos de conflicto trágico (por inconmensurabilidad o por empate entre valores) y aquellos casos en que tal situación no se da ${ }^{44}$. Nótese que la exigencia para el realismo/irenismo también funciona inversamente: se le exige que provea algún criterio que nos permita conservar la intuición de que existen DI; pero también será necesario proveer algún criterio que nos permita conservar la idea de que existen casos en los que no hay conflicto. Desde este punto de vista, una teoría que afirmara que todos los casos son casos de conflicto trágico no sería satisfactoria por las razones inversamente opuestas a las del irenismo/realismo: dejaría desamparados a quienes pensaran que, además de casos de conflicto trágico o DI en que ninguna de las partes ha cometido un error, hay casos en que no hay conflicto trágico o si lo hay será aparente y nos inclinaremos por decir que una de las partes ha cometido un error. Pero este no es el caso del pluralismo dilemático: esta hipótesis de reconstrucción contiene una explicación para los casos en que no hay conflicto ni DI y también contiene una explicación para los casos en que sí hay DI o conflicto trágico. Es decir, provee un criterio que nos permite distinguir una tesitura de la otra, algo que los críticos del realismo semántico han reclamado a este último.

En cuarto lugar, el pluralismo dilemático está en condiciones de proveer una respuesta a las dificultades con las que el relativismo indexical se encontraba en materia de gusto. Recordemos en qué consistía precisamente la objeción a la que el relativismo indexical no podía responder. En el ámbito del gusto, podemos inclinarnos por pensar que un caso en el que Riccardo prefiere el Barolo y Paolo el Priorat será un caso

ítems son inconmensurables cuando no tenemos una unidad de medida común que nos permita ordenarlos cardinalmente; pero hay una segunda manera, más amplia, de entender el término, según la cual dos ítems son inconmensurables cuando tampoco es posible una ordenación ordinal. En el caso del primer sentido hay espacio para la conmensurabilidad no-cardinal; en el segundo sentido, no hay espacio para ningún tipo de conmensurabilidad. Cuando se habla de valores prácticos (ya sean morales o bien jurídicos), se ha interpretado que incomensurabilidad quiere decir que no hay unidad de medida común que permitan ordenarlos ni cardinal ni ordinalmente. Así es como cabe entenderlo aquí.

44 Obviamente, cómo sabemos que nos encontramos o no delante de un caso trágico —a saber, cuándo dos valores son inconmensurables o están empatados- es una cuestión relevante de la que depende, en parte, que el pluralismo dilemático pueda superar la dificultad que el irenismo/realismo no podía superar. Pero, en todo caso, ésta es una cuestión perteneciente al campo de la epistemología moral. Y este trabajo no se ocupa de los problemas relativos al conocimiento moral, sino de la estructura — de los problemas conceptuales- de los sistemas prácticos. 
meramente «intachable», a saber, un caso en el que ninguno de los dos ha cometido un error y en el que el desacuerdo es aparente, puesto que cada uno de ellos apela a su propio estándar de gusto. Sin embargo, una explicación para la discrepancia entre Pierluigi, que prefiere el vino de la marca blanca del supermercado, y Riccardo y Paolo, que prefieren el Barolo al vino que propone Pierluigi, no puede consistir en decir que Pierluigi está apelando a sus propios estándares de gusto. Más bien parece que hay alguna especie de estándar común; estándar, por otra parte, que nos permitiría decir que Pierluigi está cometiendo un error y que un desacuerdo como el que él tiene con Riccardo y Paolo involucra alguna clase de error.

Pues bien, trasladado a los desacuerdos jurídicos el pluralismo dilemático está en condiciones de dar cuenta de un caso de este tipo. Afirma el pluralismo dilemático que habrá casos en que ninguna de los dos partes (o un mismo agente en caso de que se trate un conflicto trágico intra-subjetivo) habrá cometido un error: los casos de DI. Este sería el equivalente al caso de desacuerdo entre Riccardo y Paolo. Pero el pluralismo dilemático también afirma que, aunque los jueces sostengan una multiplicidad de criterios de interpretación, también existen consideraciones jurídicas erróneas. Hay ocasiones en las que podemos decir que la respuesta jurídica a un caso es errónea, ya que el derecho estará determinado (el caso paradigmático sería el de los contratos, matrimonio, etc.). Esto último equivaldría al juicio de gusto formulado por Pierluigi en el caso de los vinos: en este caso, el gusto estaría determinado y no todo dependería de los criterios subjetivos de los agentes.

En otras palabras: la ventaja del pluralismo dilemático frente al relativismo indexical y frente al escepticismo es que la validez de los criterios de interpretación (o del gusto) no siempre es relativa a (un estándar de gusto concreto o subjetivo, en el caso del primero, a los criterios subjetivos del intérprete del derecho, en el caso del segundo). Existen criterios que nos permiten hablar de al menos cierto grado de determinación en cuestiones de gusto o de derecho.

El pluralismo dilemático, así pues, constituye una vía intermedia entre los dos extremos representados por los binomios irenismo/realismo semántico y relativismo indexical/escepticismo. Subsana las ausencias del primer binomio (admite DI) y da cabida a un cierto grado de determinación en el derecho (no todo depende del estándar subjetivo del intérprete del derecho).

A modo de comentario final, quisiera hacer una observación. El pluralismo dilemático es afín en algunos aspectos, a una posición hartiana o intermedia en materia de teoría de interpretación del derecho. Como es sabido, HART ${ }^{45}$ delineó dos posiciones iusfilosóficas extremas: el noble sueño y la pesadilla. La primera estaba caracterizada por la esperanza de que los jueces nunca crearan derecho ya que existía siempre una respuesta correcta para todos y cada uno de los casos. La segunda en cambio estaba atravesada por una buena dosis de realismo — cinismo, dirían algunos- y afirmaba que los jueces siempre crean derecho ya que los casos jurídicos no tienen una respuesta objetivamente correcta. En medio de ambas, se encontraba la posición hartiana, para la cual había un núcleo de casos claros que tenían una respuesta correcta; mientras que otros casos, debido —entre otras cosas - a la textura abierta del lenguaje, eran

${ }^{45}$ HART, 1961: cap. VII; id., 1977. 
susceptibles de una pluralidad de respuestas y el juez tenía discrecionalidad para elegir entre ellas. El pluralismo dilemático es, en este sentido, afín a la teoría intermedia. Pero pone énfasis en algo que no fue destacado por HART y que sin embargo su teoría poseía: una posición intermedia explica más que sus teorías rivales. La razón por la que preferiremos una teoría intermedia, como el pluralismo dilemático, descansa en que es más consiliente ${ }^{46}$, tiene más capacidad explicativa; consigue dar cuenta de la práctica que estudia de manera más amplia porque admite, en su marco teórico, dos tipos de supuestos o fenómenos (situaciones de DI y situaciones «armónicas»).

\section{BIBLIOGRAFÍA}

AleXY, R., 1986: Teoría de los derechos fundamentales, Madrid, Centro de Estudios Políticos y Constitucionales, 1993.

BAYÓN, J. C., 1991: La normatividad del Derecho: deber jurídico y razones para la acción, Madrid, Centro de Estudios Políticos y Constitucionales.

Crowder, G., 2004: Isaiah Berlin. Liberty and Pluralism, Cambridge, Polity.

Dworkin, R., 1985: A Matter of Principle, Cambridge, Harvard University Press.

- 1986: Law's Empire, Harvard, Belknap Press.

- 2001: «Do Liberal Values Conflict?», en M. LiLLA, R. Dworkin, y R. B. Silvers (eds.), The Legacy of Isaiah Berlin, New York, NYRB.

FERRER BeltrÁn, J., 2012: «El error judicial y los desacuerdos irrecusables en el derecho», en P. LuQue, y G. B. RatTi (eds.), Acordes y desacuerdos. Cómo y por qué los juristas desacuerdan, Madrid, Marcial Pons.

GUaSTinI, R., 2009: Nuovi studi sull'interpretazione, Roma, Aracne.

HampShire, S., 2000: Justice is conflict, Princeton, Princeton University Press.

HART, H. L. A., 1961: The Concept of Law, Oxford, Oxford University Press, $2^{\text {nd }}$ ed., 1994.

- 1977: «American Jurisprudence through English Eyes: The Nightmare and the Noble Dream», en id., Essays in Jurisprudence and Philosophy, Oxford, Oxford University Press, 1983.

IACONA, A., 2008: «Faultless or Disagreement», en M. GARCÍA CARPINTERO y M. KÖLBEL (eds.), Relative Truth, Oxford, Oxford University Press.

JaHABENGLOO, R., 1990: Conversations with Isaiah Berlin, trad. cast. de M. CoHEN, por la que se cita, Conversaciones con Isaiah Berlin, Barcelona, Anaya\&Muchnik, 1996.

KöLbel, M., 2003: «Faultless Disagreements», en Proceedings of the Aristotelian Society, 104 (1), 53-73.

LeITER, B., 2009: «Explaining Theoretical Disagreements», en The University of Chicago Law Review, 1215-1250.

MoRESO, J. J., 2002: «Sobre revisiones estables, casos paradigmáticos e ideales regulativos», en id., La Constitución: modelo para armar, Madrid, Marcial Pons, 2009.

RATTI, G., 2008: «Los desacuerdos jurídicos en la jurisprudence anglosajona», en Analisi e diritto, 301-331.

Ross, A., 1958: On Law and Justice, Steven\&Sons Limited.

46 Esta característica de la teoría hartiana ha sido también destacada por B. LEITER al abordar el desafío planteado por R. DwORKIN en Law's Empire al positivismo jurídico de inspiración hartiana, vid. LEITER, 2009. Respecto a la virtud de la consiliencia, vid. THAGARD, 1978: 79. 
ShapIRO, S., 2007: «The Hart-Dworkin Debate. A Short Guide for the Perplexed», en A. RIPSTEIN (ed.), Ronald Dworkin, Cambridge, Cambridge University Press.

Thagard, P., 1978: «The Best Explanation: Criteria for Theory Choice», en Journal of Philosophy, vol. 75, n. 2, 76-92.

Wright, C., 2001: «On Being on a Quandary. Relativism, Vagyeness, Logical Revisionism», en Mind, vol. 110, 45-98. 